association between personal protective equipment (PPE) use and workplace hygiene and symptoms of neurotoxicity in vehicle spray painters.

Methods Exposure controls including PPE-use and workplace hygiene practices and symptoms of neurotoxicity were assessed in 267 vehicle repair spray painters. Symptoms were assessed using the EUROQUEST questionnaire.

Results Frequent respirator and glove use was inversely associated with symptoms of neurotoxicity in a dose-dependent manner $(\mathrm{p}<0.05$ for trend). The strongest protective effect was found for consistent glove use (OR 0.1 and 0.2, $\mathrm{p}<0.01$, for reporting $\geq 10$ and $\geq 5$ symptoms, respectively). A doseresponse trend $(\mathrm{p}<0.05)$ was also observed when combining frequency of respirator and glove use, with an overall reduction in risk of $90 \%$ (OR 0.1 for reporting $\geq 10$ and $\geq 5$ symptoms, $\mathrm{p}<0.01)$ for those who consistently used both. Protective effects were most pronounced for psychosomatic $(\mathrm{p}<0.05$ for trend, for combined PPE use), mood $(\mathrm{p}<0.05)$ and memory and concentration symptoms combined $(\mathrm{p}<0.05)$, with reductions in risk of $>80 \%$. Poor hygiene workplace practices, e.g. solvent exposure to multiple body parts were associated with an increased risk of symptoms, but this was not statistically significant. When using a general workplace hygiene score derived from a combination of PPE-use and (good) workplace hygiene an inverse dose-response trend was observed for reporting $\geq 5 \quad(\mathrm{p}<0.01)$ and $\geq 10$ symptoms $(\mathrm{p}<0.01)$.

Conclusions This study has shown that PPE-use and good workplace hygiene are associated with a strongly reduced risk of symptoms of neurotoxicity in solvent-exposed vehicle spray painters. Glove use was most protective, suggesting dermal exposures may be of particular importance in the development of symptoms.

\section{O2D.4 BENZENE AND TOLUENE EXPOSURE AND SYMPTOMS AFFECTING WORK ABILITY AMONG THE ROAD SWEEPERS IN POLLUTION CONTROL AREA, RAYONG PROVINCE, THAILAND}

Srirat Lormphongs*. Department of Industrial Hygiene and Safety, Faculty of Public Health, Burapha University, Chonburi Province, Thailand, Chonburi Province, Thailand

\subsection{6/OEM-2019-EPI.50}

Background Road sweepers exposed to the exhausted smoke and carbon deposit from the vehicles which will stimulate the allergic from various chemicals and affected to their health. The objectives were to evaluate benzene and toluene exposure and symptoms and work ability among the road sweepers in pollution control area, Rayong province, Thailand.

Methods The methods used Organic Vapor Monitor (3M 3500) for collecting air samples adhered to the road sweepers at the level of breathing, and the collection of urine in $20 \mathrm{cc}$. plastic bottles.

Results The mean age of the study group was 44.05 years. $92.0 \%$ of the study group worked 8 hours per day; $63 \%$ worked, 6 days per week. $57.0 \%$ always used respiratory protection; however, most of them used only cotton masks (97.9\%). Most of them about work ability had good level $(64.0 \%)$. Results of the study group $(n=100)$ showed average exposure measures of benzene of 20.63 ( $\mathrm{SD}= \pm 1.363 \mathrm{ppb}$ ), and toluene 86.09 ( $\mathrm{SD}= \pm 84.016 \mathrm{ppb})$. Results of urine samples $(n=100)$, after the work shift, showed average \pm SD of toluene to be $0.001 \pm 0.002 \mu \mathrm{g} / \mathrm{L}$. The average comparison of concentration of benzene and toluene was significantly different between the study and comparison groups at level 0.05 $(\mathrm{p}<0.001$ and $\mathrm{p}<0.001$, respectively). However, the relationship between benzene and toluene in the atmosphere and in the urine and work ability of study group were not significant.

Conclusion Based on the results of this study, benzene and toluene in the atmosphere and in the urine and symptoms among road sweepers were detected. Health promotion and protection programs should be emphasized. Respiration protective equipment should also be provided.

\section{O2D.5 RISK OF PARKINSON DISEASE IN SOLVENT EXPOSED WORKERS IN FINLAND}

Markku Sallmén*, Sanni Uuksulainen, Christer Hublin, Aki Koskinen, Markku Sainio. Finnish Institute of Occupational Health, Helsinki, Finland

\subsection{6/OEM-2019-EPI.51}

Epidemiologic studies indicate that occupational exposure to solvents may increase risk of Parkinson disease (PD).

We constructed a population-based case-control study of incident PD using a register of Reimbursement of medicine costs of the Social Insurance Institution of Finland, along with the Population Information System, including census records for all Finnish residents. PD cases were diagnosed between 1995-2014. Controls were randomly selected from the population while matching on diagnosis year, birth year (1930-1950), and sex. A total of 11,757 PD cases and 23236 controls had data from the occupational census in 1990, ensuring $\geq 4$ years exposure lagging and 21 years of occupational history data $(5$ censuses from 1970-1990). We used the Finnish Job Exposure Matrix to assess cumulative exposure (CE) to four groups of solvents (aliphatic/alicyclic hydrocarbon, aromatic hydrocarbon, chlorinated hydrocarbon, other). We estimated PD-solvent odds ratios (ORs) and 95\% confidence intervals (CIs) using unconditional logistic regression, while adjusting for age, sex, socioeconomic status and smoking (a_OR), or additionally for CE to chromium and one of the other solvent groups (ab_OR).

In total, 3758 cases $(30.4 \%)$ and 7445 controls $(32.0 \%)$ were potentially exposed to solvents (a_OR 0.99; CI: 0.94-1.05). Exposure to chlorinated hydrocarbons was associated with PD (a_OR 1.20; CI: 1.05-1.36; ab_OR 1.21 CI: $1.04-1.40)$ at the highest CE group (20-145 ppm-years, $n=409$ cases and 728 controls) but not at lower CE levels. Overall, CE to chlorinated hydrocarbons $(n=1840$ cases and 3693 controls) was associated with increased risk of PD ( $\mathrm{p}$-for-trend $=0.01)$. There was no evidence of a positive association for any of the other solvent groups.

We observed a positive association between occupational exposure to chlorinated hydrocarbons and risk of PD. This was especially true for greatest duration and/or level of exposure. 